\title{
Etude de la dynamique des populations d'insectes sur la culture du riz NERICA dans les conditions du Masuku, Sud-Est du Gabon (Franceville)
}

\author{
Paul ONDO OVONO ${ }^{1 *}$, Thaddée GATARASI ${ }^{1}$, Daniel OBAME MINKO ${ }^{2}$, \\ Drice MIYOUMBI KOUMAGOYE ${ }^{1}$ et Claire KEVERS ${ }^{3}$
}

\author{
${ }^{1}$ Unité de Recherche Agrobiologie, Institut National Supérieur d'Agronomie et de Biotechnologies, \\ Université des Sciences et Techniques de Masuku, B.P. 941, Masuku, Gabon. \\ ${ }^{2}$ Ecole Normale Supérieure de Libreville, BP 17009 Libreville, Gabon. \\ ${ }^{3}$ Plant Molecular Biology and Biotechnology Unit, Sart Tilman, B-22, University of Liège, B- 4000 Liège, \\ Belgium. E-mail: c.kevers@ulg.ac.be, Fax: + $32(0) 43363846$ \\ *Auteur correspondant, E-mail: paulondo@hotmail.com ; Tel/Fax : + (241)671334/35
}

\section{RESUME}

La dynamique des populations d'insectes sur la culture du riz a été étudiée durant toutes les phases de croissance. L'essai a été installé sur les parcelles pédagogiques de l'Institut National Supérieur d'Agronomie et de Biotechnologies à Franceville. Il s'inscrit dans le cadre de la poursuite des travaux d'évaluation de l'adaptabilité de nouvelles variétés de riz de type NERICA, introduites dans la province du Haut -Ogooué au Sud-Est du Gabon. L'objectif de la présente étude est de collecter des informations concernant les insectes susceptibles de s'attaquer au riz et de sélectionner les variétés NERICA qui résistent le mieux aux attaques. Chacune des six variétés de type NERICA $(1,4,11,12,14$ et 17) a été semée en utilisant un dispositif en blocs complètement randomisés avec trois répétitions, en 2010 et 2011. La collecte des insectes volants a été effectuée au moyen de pièges aériens et du filet entomologique. Au sol, la capture s'est faite au moyen de pièges. Les larves d'insectes foreurs des tiges, les chenilles et les pucerons des racines ont été capturés à la main. Au total, 46 familles appartenant à 9 ordres ont été recensées donc 29 renferment des espèces nuisibles et définies comme ravageuses du riz. Les 17 autres familles sont classées comme insectes utiles. Les stades de tallage, d'initiation paniculaire et d'épiaison ont été en général les plus visités par les différentes familles d'insectes. Les variétés NERICA 1 et NERICA 11 ont présenté les plus faibles taux d'infestation et de dégâts. Par ailleurs, la perte en biomasse a été considérable chez toutes les variétés.

(C) 2014 International Formulae Group. All rights reserved.

Mots clés: Riz pluvial, NERICA, variétés, ordres et familles d'insectes, Franceville, Gabon.

\section{INTRODUCTION}

Le riz est une monocotylédone de la famille des Poacées. Il appartient à la tribu des Oryzées qui regroupe un nombre de genres variables selon les classifications compris entre 7 et 16 (Schalbroeck, 2001; Courtois,
2007). Les données taxonomiques les plus récentes considèrent que le genre Oryza compte lui-même 24 espèces dont deux sont cultivées, Oryza sativa L. à distribution mondiale et Oryza glaberrima Steud., 
cantonnée à l'Afrique de l'Ouest (IRRI, 2005).

$\mathrm{Vu}$ l'importance agronomique et économique que revêt la culture du riz dans le monde, des centres de recherches ont été mandatés pour mener des recherches sur le riz: l'IRRI (International Rice Research Institute) aux Philippines, à l'échelle mondiale et plus spécialement pour l'Asie; le CIAT (Centro Internacional de Agricultural Tropical) en Colombie pour l'Amérique latine; ADRAO (Association pour le Développement de la Riziculture en Afrique de l'Ouest; et l'IPGRI ( International Plant Genetic Resources Institute, en France) dans le domaine des ressources génétiques (Ndayiragije, 2006).

Au Gabon, le riz fait désormais partie intégrante des habitudes alimentaires de la population. La consommation moyenne en 2009 était estimée à près de 70.000 tonnes entièrement importées (Nguimbi, 2004). La dépendance du Gabon vis-à-vis des besoins en riz étant de $100 \%$, seule une production locale permettrait de réduire les importations qui grèvent son budget de 250 milliards de francs CFA chaque année. L'Etat gabonais, par l'entremise de l'Office National du Développement Rural (ONADER) en partenariat avec Africa Rice et la coopération japonaise, a décidé d'introduuire de nouvelles variétés de riz pour la relance des activités rizicoles au Gabon (Dogbe, 2009).

Le nouveau riz pour l'Afrique ou NERICA, de type pluvial, répond favorablement à une grande plasticité vis-à vis de ses conditions (Akintayo et al., 2008). Il a été obtenu par croisements interspécifiques en vue de combiner la productivité du riz asiatique, $O$. sativa et la hardiesse du riz africain, $O$. glaberrima (ADRAO, 2003). Ces variétés NERICA ont fait l'objet d'évaluation et de tests de sélection variétale participative (PVS) dans la province de l'Estuaire au Gabon. Actuellement, la sélection variétale participative se poursuit avec les variétés de riz pluvial dans plusieurs autres sites notamment à Franceville au SudEst du Gabon (Maganga, 2010).

Toutefois, le riz pluvial est généralement la proie de maladies et d'attaques de ravageurs, notamment les insectes, les nématodes, les rongeurs, les oiseaux et autres animaux. D'après les travaux réalisés à l'IRRI ainsi qu'à l'IRD, parmi les facteurs biotiques les plus importants qui sont responsables de la chute de la productivité du riz dans le monde, les adventices sont de loin les plus nuisibles aux cultures, suivies par ordre décroissant des champignons, des insectes et des bactéries. Parmi les facteurs abiotiques qui limitent le plus les rendements, on peut citer la température, la toxicité et les carences en nutriments ainsi que la salinité (Ndayiragije, 2006). Lors de ses travaux sur l'évaluation au champ des performances agromorphologiques des variétés de riz NERICA cultivées au Sud-Est du Gabon, Maganga (2010) a signalé la présence de plusieurs insectes s'attaquant à la culture. Pour mieux évaluer l'impact de cette contrainte sur les nouvelles variétés de riz, il fut nécessaire de connaître l'importance des ennemis en présence. Le présent travail a pour but d'inventorier les principaux insectes susceptibles de s'attaquer à la culture du riz pluvial dans les conditions de Masuku (Franceville) et de spécifier les variétés de riz de type NERICA qui tolèrent le mieux ces attaques.

\section{MATERIEL ET METHODES Matériel végétal}

Le matériel végétal utilisé pour la mise en place de l'essai est composé de six variétés de riz de type NERICA $(1,4,11,12,14,17)$, fournies par l'Office National du Développement Rural. Ce sont des variétés de riz pluvial, hybrides dont la maturation varie entre 90 et 100 jours. Le rendement potentiel de ces variétés varie selon les zones de culture entre 4,5 et 7t/ha (Akintayo et al., 2008). 


\section{Site de l'étude}

La zone d'étude est située dans le département de la Mpassa, province du HautOgooué. La région s'inscrit grossièrement dans un rectangle dont les coordonnées sont $13^{\circ}$ et $14^{\circ} 20$ Est, et $1^{\circ}$ et $1^{\circ} 45^{\prime}$ Sud. L'altitude moyenne est comprise entre 300 et $600 \mathrm{~m}$. Le climat de la zone d'étude est de type équatorial de transition, comportant deux saisons bien marquées. Une grande saison sèche (de mai à septembre) et une grande saison de pluie (mars - mai). Entre ces saisons sont intercalées deux saisons sèches et pluvieuses. Les précipitations moyennes annuelles sont de l'ordre de 1700 à $2200 \mathrm{~mm}$. Elles sont réparties sur toute l'année avec des variations. Les températures moyennes annuelles sont de l'ordre de $24,6{ }^{\circ} \mathrm{C}$. Les mois de mars $\left(25,1{ }^{\circ} \mathrm{C}\right)$, avril - mai $\left(25,4{ }^{\circ} \mathrm{C}\right)$ sont les plus chauds. Les sols de plateaux et plaines, les seuls utilisés dans cette étude, sont de type ferralitique fortement désaturés. Le pH du sol, toujours acide, varie de 4,5 à 5 et la teneur en argile est assez importante (45-50\%) (Itongo, 1998). Dans cette zone, on y rencontre une formation de savane anthropique. Sur les plateaux et les collines, la végétation est pauvre et herbacée (Figure 1).

\section{Méthodes}

L'étude a été conduite de 2010 à 2011 sur les parcelles pédagogiques de l'Institut National Supérieur d'Agronomie et de Biotechnologies (INSAB). Les données collectées sur le terrain ont été analysées au laboratoire de phytoprotection de l'INSAB.

\section{Mise en place de l'essai en plein champ Préparation du terrain}

Le terrain expérimental a été défriché, dessouché et labouré manuellement à plat. Chaque parcelle élémentaire mesurait $4 \mathrm{~m}$ de long sur $1 \mathrm{~m}$ de large. La superficie totale utile est de $72 \mathrm{~m}^{2}$. Les allées sont de $1 \mathrm{~m}$ entre les blocs et de $0,5 \mathrm{~m}$ entre les parcelles élémentaires (Figure 2), soit une superficie totale de l'essai de $203 \mathrm{~m}^{2}$ ( $29 \mathrm{~m} \mathrm{x} 7 \mathrm{~m}$ ).

\section{Fertilisation}

Après le labour et l'affinage, un épandage de fiente de poule a été effectué à raison de 30t/ha, soit $12 \mathrm{~kg}$ par parcelle de $4 \mathrm{~m}^{2}$. L'engrais NPK (15-15-15) a été épandu comme fumure de fond à la dose de $200 \mathrm{~g}$ par parcelle (500 kg/ha).

\section{Dispositif expérimental}

Le dispositif expérimental pour l'essai de la culture est de type blocs complètement randomisés à six variétés de riz NERICA réparties en trois répétitions (Figure 2). Chaque bloc est divisé en six parcelles élémentaires. Chaque parcelle élémentaire est formée de 5 lignes de semis ayant chacune 19 poquets, soit au total 95 poquets par parcelle. Le semis a été effectué le 26 juillet 2010 pour le premier essai et le 06 juin 2011 pour le second, à raison de 5 graines par poquet aux écartements de $20 \mathrm{~cm}$ x $20 \mathrm{~cm}$, et à une profondeur de $3 \mathrm{~cm}$.

En raison des exigences en eau du riz, une quantité de 22 litres était apportée chaque jour (au moyen d'arrosoirs) par parcelle pendant tout le cycle végétatif. Sept sarclobinages ont été effectués, à la main, tout au long du cycle cultural, avec deux apports d'urée comme fumure d'entretien au quatrième et au sixième sarclo-binage à une dose de $200 \mathrm{~kg}$ par ha. Le premier sarclobinage a eu lieu 11 jours après le semis. Les autres passages ont été effectués, respectivement les $21,35,45,69,73$ et 87 jours après le semis. Durant toute la période de l'essai, en dehors des sarclages manuels et de la fertilisation minérale, aucun traitement phytosanitaire n'a été appliqué à la culture du riz.

\section{Méthodes de collecte des insectes}

Afin de maximiser les données entomologiques sur la végétation du riz, il a été nécessaire d'utiliser plusieurs méthodes d'échantillonnage qui vont du piégeage à la capture au filet fauchoir et à la main. Selon Kumar (1991), un nombre élevé d'échantillons permet de justifier l'exactitude 
des résultats obtenus. Ainsi, chaque stade phénologique du riz a été échantillonné durant tout le cycle cultural. Chaque méthode d'échantillonnage est matérialisée par deux lettres :

- $\quad$ PS : pièges au sol ;

- $\mathrm{AJ}$ : pièges aériens aux assiettes (ou bacs) jaunes ;

- $\quad$ CF : capture au filet fauchoir ;

- $\quad \mathrm{CM}$ : capture à la main.

Chaque boîte de Pétri contenant les insectes collectés sur le terrain était marquée par une référence indiquant: la méthode de collecte, le numéro du bloc, la date et la variété du riz.

\section{Pièges au sol}

La technique consiste à piéger les insectes circulant dans et sur le sol à l'aide de 72 bocaux de $200 \mathrm{ml}$ chacun, remplis au 2/3 d'eau savonneuse (pour retenir les insectes piégés) et répartis à raison de 4 pièges par parcelle. Ces bocaux ont été déposés deux semaines après le semis, dans des trous équidistants de $1 \mathrm{~m}$ et creusés le long de la ligne centrale de culture, le bord du bocal affleurant le sol. La collecte des insectes piégés se faisait tous les lundis et jeudis à l'aide d'une mini passoire. L'eau était renouvelée une fois par semaine.

Pièges aériens (bacs jaunes)

Dix huit bacs jaunes (piège jaune FLORA avec tuteur $1.50 \mathrm{~m}$ de hauteur) remplis de l'eau savonneuse ont été placés chacun au milieu de chaque parcelle à une hauteur de $1 \mathrm{~m}$. Ces pièges ont l'avantage d'attirer les insectes circulant sur les parties aériennes des plantes. Selon Dupriez et al., (2001), la couleur jaune est reconnue pour son attraction sur la plupart des insectes volants. Ces pièges ont été installés un mois après le semis. La collecte se faisait une fois par semaine à l'aide de la mini passoire et d'une pince métallique. Afin de conserver les insectes piégés jusqu'à la prochaine collecte, dix grammes de sel de cuisine ont été ajoutés (Limoges, 2003) dans l'assiette chaque fois lors du renouvellement de l'eau savonneuse.

\section{Collecte au filet fauchoir}

La collecte au filet entomologique a été faite une fois par semaine. Cette technique consiste à effectuer un mouvement manuel rapide de gauche vers la droite et d'un bout à l'autre sur la longueur de chaque parcelle élémentaire. Les insectes capturés sont placés dans des boîtes de pétri contenant du coton imbibé d'alcool afin de les asphyxier et de pouvoir les identifier et les dénombrer ultérieurement.

\section{Collecte à la main et élevage au laboratoire}

La technique de collecte à la main a porté uniquement sur les insectes mineurs des tiges et les chenilles défoliatrices qui ne peuvent pas être capturés par d'autres méthodes disponibles. La collecte à la main a été réalisée sur toute la superficie de chaque parcelle. Cette méthode consiste à observer visuellement l'aspect morphologique des plantes et de dénombrer les tiges anormales. Une fois par semaine, les tiges perforées étaient coupées au ras du sol, comptées et transportées au laboratoire d'analyses entomologiques. Au laboratoire, les tiges sont disséquées, les larves libérées et comptées. Selon les recommandations d'Appert et Deuse (1988), le nombre de larves comptées peut être extrapolé à l'hectare. La moitié des larves sert à l'identification directe et l'autre moitié est élevée pour les besoins ultérieurs d'identification des imagos. Les tiges de riz fraîchement coupées ont servi d'alimentation aux larves.

Les chenilles défoliatrices observées dans des feuilles enroulées en forme de tube, ou simplement sur les feuilles étaient comptées, séparées selon leur morphologie externe et décrites. La moitié de ces chenilles était ensuite placée dans des bocaux d'élevage et alimentée tous les deux jours à l'aide des feuilles fraîches de riz. Les bocaux d'élevage étaient nettoyés une fois par semaine.

Les adultes des lépidoptères qui émergeaient étaient mis dans une cage d'élevage et étaient alimentés à l'eau sucrée avant de les identifier. 


\section{Identification des insectes}

La clef de reconnaissance des familles de Delvare et Aberlenc (1989) a été utilisée pour l'identification des insectes récoltés. Nous nous sommes également servis des orientations données par Appert et Deuse (1988) et Boucher (2008).

Autres paramètres

- L'observation hebdomadaire des touffes de riz détruites par d'autres ravageurs ;

- L'observation et le dénombrement ( par parcelle) de touffes présentant un retard de croissance imputable aux pucerons des racines ;

- L'observation et le dénombrement des pucerons sur les racines du riz, en déracinant les touffes présentant un retard de croissance. Cette opération était effectuée une fois par semaine à partir de 70 jours après le semis (date de la découverte des pucerons sur les racines), sur 5 poquets par parcelle. Les pucerons prélévés à l'aide du pinceau étaient placés dans un tube contenant de l'alcool et acheminés vers le laboratoire pour l'indentification ultérieure ;

- Le dénombrement des poquets ayant atteint la maturité par parcelle et par variété ;

- L'observation avant la récolte sur 5 panicules choisies au hasard et en zigzag par parcelle (Tréca et al., 1997) en vue de vérifier l'état des grains suite aux attaques d'oiseaux et d' insectes.

- L'évaluation du taux d'infestation du riz selon la population d'insectes ravageurs accumulés durant le cycle cultural de chaque variété.

\section{Récolte}

Le riz a été récolté le 17 septembre 2011 pour le second essai, au $104^{\mathrm{e}}$ jour après le semis lorsque les panicules présentaient déjà une couleur paille. Cette récolte a été effectuée en coupant les touffes à $7 \mathrm{~cm}$ audessus du sol. Les tiges étaient rassemblées en bottes, numérotées parcelle par parcelle et acheminées vers le laboratoire en vue de déterminer la biomasse.

$\mathrm{Au}$ laboratoire, les paramètres suivants ont été mesurés :

- Poids de la biomasse végétative aérienne (fraîche) récoltée ;

- Nombre de tiges récoltées par parcelle ;

- Poids de la biomasse aérienne des tiges indemnes de dégâts (imputables aux attaques d'insectes et d'oiseaux).

Le niveau d'infestation des foreurs des tiges Taux d'infestation des foreurs $(\%)=($ Nombre de tiges infestées / Nombre total de tiges) $\mathrm{x}$ 100 .

\section{Niveau d'infestation des pucerons des racines}

Pourcentage $(\%)$ de touffes chétives $(\mathrm{TC})=$ (Nombre de touffes chétives/ Nombre total de touffes) x 100 .

La perte en biomasse végétative aérienne fraîche a été déterminée par la relation suivante :

$\mathrm{P} 2=\mathrm{P} 0-\mathrm{P} 1$

$\mathrm{P} 0$ : poids total des tiges à la récolte ;

$\mathrm{P} 1$ : poids des tiges indemnes ;

$\mathrm{P} 2=$ pertes.

$\%=(\mathrm{P} 2 / \mathrm{P} 0) \times 100$

$\%$ : pourcentage de pertes.

\section{Analyse statistique}

Les données relatives au nombre total d'insectes ravageurs, au niveau d'infestations des foreurs des tiges, des chenilles défoliatrices, des pucerons des racines et du pourcentage de perte de la biomasse végétative aérienne ont été soumises à l'analyse de la variance à l'aide du logiciel XLSTAT-2007. En cas de différence, les moyennes étaient séparées par le test de DUNCAN au seuil $5 \%$. 


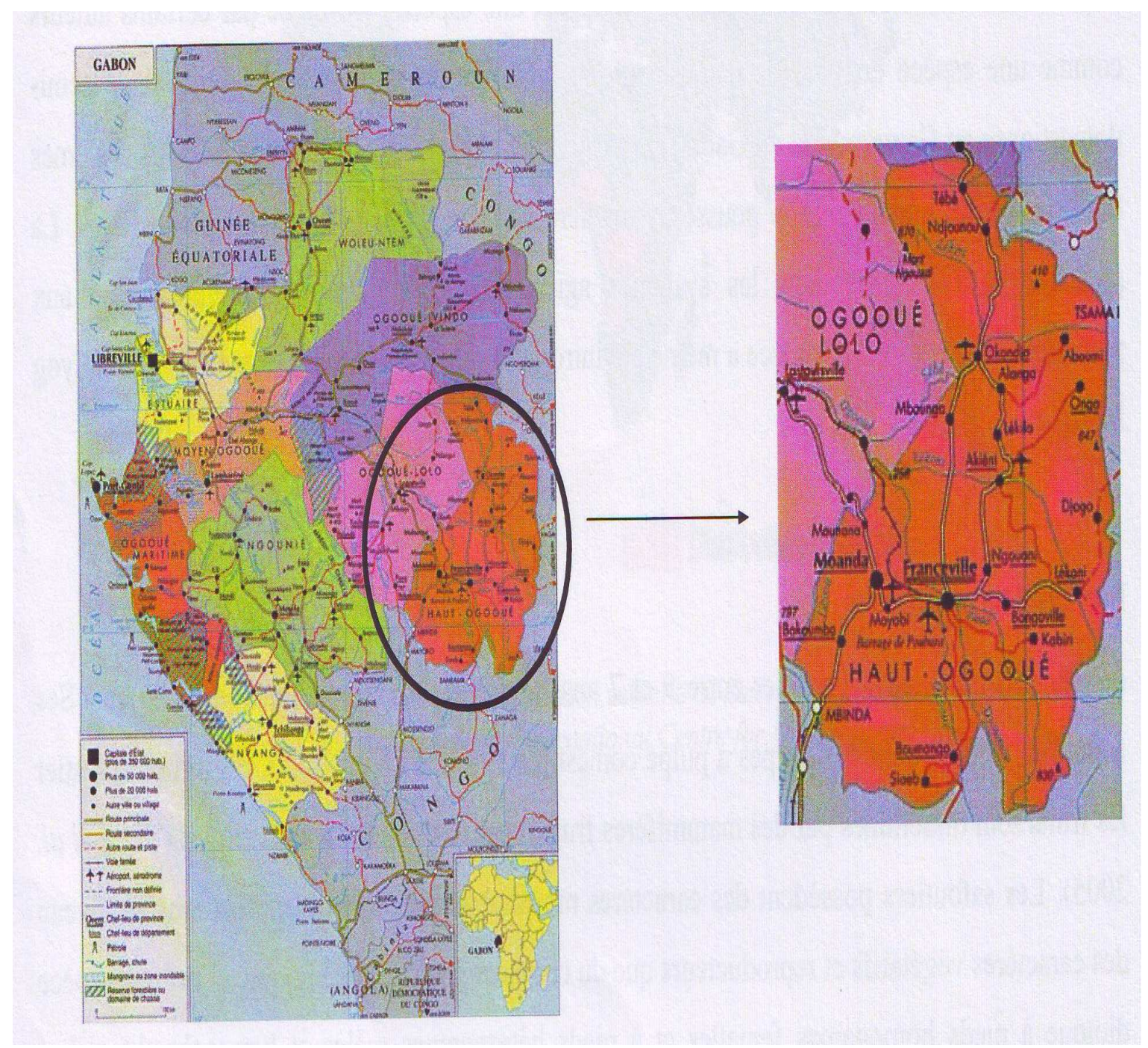

Figure 1 : Localisation de la région et de la zone d'étude (Anonyme, 2003).

\section{RÉSULTATS ET DISCUSSION}

Inventaire des insectes collectés avec les pièges au sol (PS), les bacs jaunes (AJ) et le filet entomologique (CF)

Le nombre et le pourcentage d'insectes capturés par ordre, famille et par piège durant tout le cycle végétatif de chacune des variétés du riz NERICA sont présentés dans le Tableau 1. Au total, 46 familles appartenant à 9 ordres d'insectes ont été collectées sur les variétés de riz NERICA. La plus forte population se trouve dans l'ordre des Hémiptères (395 individus), soit $41,19 \%$ des insectes collectés. Les Orthoptères (218) viennent en second lieu avec 22,73\%, suivis des Diptères (123) soit $12,83 \%$, des Lépidoptères (93) soit 9,70\%, des Hyménoptères (64) soit 6,67\%, des Coléoptères (42) soit 4,38\%, des Thysanoptères (11) soit $1,15 \%$, des Dictyoptères (8) soit $0,83 \%$ et enfin des Névroptères (5) soit $0,52 \%$.

Les Orthoptères ont été abondamment collectés à l'aide des pièges du sol alors que les Hyménoptères, Diptères, Lépidoptères et 
les Hémiptères l'ont été dans les pièges aériens. Les Coléoptères ont été collectés principalement à l'aide du filet. Les Névroptères et les Thysanoptères ont été uniquement collectés respectivement au moyen des pièges aériens et $\mathrm{du}$ filet entomologique.

Les insectes collectés sur les parcelles de riz NERICA à Franceville sont présents aussi bien au niveau du sol que sur les parties aériennes de la culture et chaque type de piège utilisé s'accommode bien à un ordre d'insectes défini. Parmi les ordres collectés, six (Hémiptères, Orthoptères, Diptères, Lépidoptères, Coléoptères et Thysanoptères) renferment des individus susceptibles d'être nuisibles à la culture du riz. A ces six ordres, on peut ajouter dans une moindre mesure l'ordre des Hyménoptères avec la famille des Formicidae dont certains individus peuvent indirectement être considérés comme ennemis de la culture à cause de leur rôle symbiotique de protection des insectes producteurs de miellat tels que les pucerons (Pande et al., 1997 ; Michel et Bournier, 1997) et à cause des galeries qu'ils creusent autour des touffes de riz, fragilisant ainsi l'enracinement des plants. Toutefois, nous n'avons pas classé cette famille dans la catégorie des insectes ravageurs car lors de notre essai, ses actions néfastes sur le riz en végétation n'ont pas été décelées.

\section{Nombre et dynamique d'insectes de l'ordre des Orthoptères}

Six familles d'insectes ravageurs ont été collectées: Gryllidae, Tettigoniidae, Thericleidae, Pyrgomorphidae, Tetrigidae et Rhipipterygidae. Ces quatre dernières familles ont été rassemblées sous l'appellation de «Sauteriaux ». Les Sauteriaux et les Gryllidae renferment des individus reconnus comme ravageurs du riz (Pande et al., 1997 ). L'étude a montré que la famille des Gryllidae ne se retrouve qu'au niveau du sol et renferme les individus les plus nombreux après les Sauteriaux. Ces derniers ont aussi été capturés à l'aide du filet sur la végétation. Ils sont les plus nombreux sur toutes les variétés avec une population supérieure à 25 individus par parcelle de $4 \mathrm{~m}^{2}$. Les Tettigoniidae n'ont été observés que dans les pièges aériens et ont aussi été capturés à l'aide du filet. Les individus de cet ordre s'attaquent à tous les organes de la plante. Les insectes appartenant à l'ordre des Orthoptères s'observent du stade de plantule aux stades de maturation du riz. Ce sont donc des ravageurs qui s'attaquent au riz durant tout son cycle végétatif. Toutefois, ils sont plus abondants aux stades de tallage et d'initiation paniculaire. Une représentativité faible et parfois nulle à partir du stade montaison, s'explique d'une part, par le fait que certains insectes comme les Gryllidae qui se nourrissent des jeunes racines ou des jeunes tiges ne sont plus présents sur les plants adultes. D'autre part, on note l'envahissement de la parcelle par les fourmis rouges, précisément dans les pièges placés au niveau du sol compromettant ainsi la collecte des autres insectes. Il est à préciser que les Orthoptères observés à partir du stade montaison appartiennent essentiellement à la famille des Tettigoniidae et aux familles regroupées sous l'appellation de Sauteriaux parmi lesquelles la famille des Pyrgomorphidae (avec l'espèce Zonocerus variegatus) est la plus dominante. Nous pouvons affirmer que, chez les Orthoptères, les sauteriaux sont les plus à craindre sur la culture de par leur nombre et leur apparition dans le temps.

\section{Nombre et dynamique d'insectes de l'ordre des Diptères}

Sept familles de l'ordre des Diptères ont été collectées dont trois (Agromyzidae, Chloropidae, Diopsidae) sont nuisibles aux 
cultures. Sur la culture du riz, seules les familles des Chloropidae et des Diopsidae renferment des individus reconnus comme étant des ravageurs (Polaszek et Delvare, 2000). La famille des Agromizidae possède un nombre d'individus plus élevé chez toutes les variétés du riz. Ils ont été collectés à l'aide des pièges au sol et aériens. C'est également le cas des individus de la famille des Chloropidae. Les individus de la famille des Diopsidae sont les moins représentatifs et de ce fait n'ont été observés que sur le NERICA 17.

Bien que les Agromizidae semblent être les plus dangereux pour la culture du riz à Franceville de par leur nombre d'individus, l'on sait que les individus appartenant aux familles des Chloropidae et des Diopsidae sont les plus à craindre en rizière, car ce sont des foreurs des tiges.

Les Diptères sont signalés sur le riz du stade de plantule au stade de maturation. C'est surtout aux stades tallage et à l'initiation paniculaire que leur présence est fortement signalée. Cette abondance peut s'expliquer par le fait que le tallage maximal se fait au début de l'initiation paniculaire, ce qui est avantageux pour les jeunes larves des Diptères (Dobelmann, 1976b).

\section{Nombre et dynamique d'insectes de l'ordre des Coléoptères}

Quatre familles de Coléoptères ont été collectées mais seule la famille des Chrysomelidae renferme des individus reconnus comme ravageurs s'attaquant aux feuilles des plants de riz ( Dobelmann, 1976a $\&$ b). Ils ont été collectés à l'aide du filet et des pièges aériens. Notre étude a montré qu'aucune variété du riz NERICA n'a échappé aux attaques des Coléoptères Chrysomèles pendant sa croissance végétative active. La présence des coléoptères est importante sur le riz aux stades tallage, initiation paniculaire, montaison et maturation, le seul stade qui présente une attaque généralisée sur toutes les variétés.

\section{Nombre et dynamique d'insectes de l'ordre des Lépidoptères}

Dans cet ordre, quatre familles d'insectes (Noctuidae, Pyralidae, Arctiidae, Nymphalidae) ont été collectées et identifiées. On constate que les insectes appartenant aux familles des Lépidoptères ont été abondamment collectés au moyen des pièges aériens. La famille des Pyralidae domine avec un maximum de près de 10 individus piégés pendant le cycle végétatif respectivement sur les variétés NERICA 4 et NERICA 14. En nombre d'individus, cette famille est suivie par les Noctuidae.

Les familles identifiées dans cet ordre renferment des individus qui, à l'état larvaire sont nuisibles aux cultures, mais seules les espèces appartenant aux familles des Noctuidae et Pyralidae sont fortement reconnues comme étant des ravageurs permanents (foreurs des tiges ou défoliateurs) dans les rizières (Polaszek et Delvare, 2000).

Les lépidoptères sont signalés sur le riz du stade tallage au stade de maturation, mais ils sont plus abondants aux stades tallage et épiaison-floraison. La présence de ces insectes adultes indique le début de la ponte et non celui des attaques. Comme pour les diptères, la fonction de ravageurs est uniquement assurée par les individus au stade larvaire et le début des attaques dépendra de la durée d'incubation des œufs pondus.

\section{Nombre et dynamique d'insectes de l'ordre des Hémiptères}

Dans l'ordre des Hémiptères, quinze familles ont été collectées dont 14 (Lygaeidae, Cicadellidae, Aphididae, Coreidae, Ricaniidae, Membracidae, Alydidae, Cercopidae, Lophopidae, Pyrrhocoridae, Scutelleridae, Plataspidae, Pentatomidae et 
Delphacidae) sont classées comme nuisibles aux cultures. Les insectes appartenant aux familles des Aphididae (plus de 50 individus par cycle et par variété de riz), des Cicadellidae, des Delphacidae et des Alydidae, largement collectés dans les pièges aériens sont particulièrement abondants. Ces familles possèdent des individus reconnus comme ravageurs de rizières. Ils piquent les différents organes du plant et en sucent la sève.

A ces insectes signalés comme ravageurs sur le riz s'ajoutent les familles des Pentatomidae et des Coreidae apparus en petits nombres dans l'essai. Des individus appartenant aux familles des Cicadellidae et Delphacidae sont en même temps cités comme vecteurs de viroses (Stoll, 2002).

La présence des Hémiptères sur le riz débute au stade de tallage et se poursuit jusqu'à la maturation avec un pic au stade d'épiaison. Cela prouve que les Hémiptères apprécient plus les organes jeunes de reproduction (épis et fleurs). Au stade de maturation du riz, la population des Hémiptères baisse, soit parce qu'il n'y a plus de nourriture en suffisance, soit parce que le nombre de leurs ennemis naturels s'est accru.

\section{Nombre et dynamique d'insectes de l'ordre des Thysanoptères}

Dans l'ordre des Thysanoptères, une seule famille a été collectée au moyen du filet. Les individus de cette famille sont reconnus comme étant des ravageurs de la culture du riz. Ils sucent la sève sur toutes les parties aériennes des plants de riz. L'analyse montre que toutes les variétés ont hébergé les thrips en petits nombres, car ces insectes apparaissent surtout pendant la floraison et il n'est pas aisé d'ouvrir les fleurs pour les retrouver.

Les individus de l'ordre des Thysanoptères ne sont apparus qu'à partir du stade d'initiation paniculaire jusqu'à l'épiaison-floraison. Leur abondance sur toutes les variétés s'observe plus précisément au stade montaison. L'absence de ces insectes au stade maturation n'est pas significative sur la culture. En effet, certains individus de cet ordre ont une préférence pour les panicules, et donc ils se retrouvent le plus souvent entre la glume et le grain en développement. Dans ces conditions, il devient difficile de les capturer.

$\mathrm{Au}$ regard de tout ce qui précède, il ressort que la plupart des insectes inventoriés font partie du relevé des familles d'insectes possédant des individus nuisibles au riz cultivé en Côte d'Ivoire, au Sénégal, en Sierra Léone, au Mali, au Bénin et au Cameroun (Brénière, 1969). Les stades de tallage, d'initiation paniculaire et d'épiaison-floraison ont été les plus visités par les insectes ravageurs. La présence et la permanence des populations d'insectes ravageurs sur la culture du riz en fonction de leur régime alimentaire spécifique déterminent le niveau de destruction de la plante (infestation et dégâts).

\section{Le niveau d'infestation}

L'analyse statistique des données sur le niveau d'infestation du riz calculé sur la base du nombre d'insectes ravageurs recensés (795) durant tous les stades végétatifs du riz (Tableau 2) montre une différence significative entre les variétés au seuil $5 \%(\mathrm{P}=$ 0,010). La densité d'insectes par variété de riz oscille entre $15 \%$ et $18 \%$ de ravageurs recensés. Les variétés les plus fréquentées par les insectes ravageurs sont NERICA 14 (18\%) et NERICA $17(18 \%)$. Les variétés NERICA $11(15 \%)$ et NERICA 12 (15\%) sont les moins fréquentées. Comparativement au nombre total d'insectes collectés (959; Tableau 1), il en ressort que $83 \%$ d'insectes sont classés comme ravageurs de la culture du riz NERICA. Les $17 \%$ restant sont répartis entre les insectes auxiliaires, polinisateurs, 
nécrophages, coprophages et saprophages. Le potentiel des insectes ravageurs $\mathrm{du}$ riz augmente avec l'extension des surfaces cultivées (Brenière, 1966).

\section{Les insectes utiles}

Tous les insectes collectés sur les parcelles expérimentales ne sont pas des ravageurs de la culture du riz. Certains d'entre eux sont des parasites, d'autres des prédateurs des insectes nuisibles. Ces derniers sont répartis dans onze familles appartenant à cinq ordres: Hémiptères (Reduviidae), Diptères (Tachinidae, Syrphidae), Hyménoptères (Elasmidae, Vespidae, Formicidae), Coléoptères (Carabidae, Coccinellidae, Lampyridae) et Névroptères (Chrysopidae, Hemerobiidae). Les individus appartenant à ces familles sont des insectes qualifiés d'auxiliaires, car ils s'attaquent à un ou à plusieurs insectes ravageurs des cultures assurant ainsi un certain équilibre au sein des parcelles agricoles (Boucher, 2008).

Spécifiquement, les Vespidae et les Reduviidae renferment des individus prédateurs des chenilles et des larves des coléoptères, alors que les individus des familles des Coccinellidae, des Chrysopidae, des Hemerobiidae et des Syrphidae sont prédateurs des pucerons. Les Elasmidae sont des hyperparasites chez les lépidoptères. Quant aux familles des Carabidae, des Lampiridae, des Formicidae et des Tachinidae, elles comptent des individus respectivement prédateurs et parasites d'insectes variés (Simon et al., 1994 ; Michel et Bournier, 1997 ). Concernant les insectes coprophages et saprophages (Calliphoridae, Stratiomyidae), ils sont aussi utiles car ils accélèrent le processus de décomposition de la matière organique et jouent donc un rôle important pour l'enrichissement du sol (Boucher, 2008).

\section{Les insectes collectés à la main}

Cette catégorie d'insectes concerne les larves d'insectes foreuses de tiges, les chenilles défoliatrices et les pucerons des racines.

\section{Larves foreuses des tiges}

Après la récolte et la dissection des tiges présentant les symptômes d'attaque par les foreurs de tiges, plusieurs types de larves ont été dénombrés et identifiés. Toutes les larves identifiées appartiennent aux ordres des Lépidoptères et des Diptères (Figure 3); une seule chrysalide a été recensée.

\section{Description des larves et évolution des dégâts}

Les larves de Diptères, foreurs de tiges, dont la taille varie entre 3 à $5 \mathrm{~mm}$ de long, sont blanches et translucides, dépourvues de pattes, la tête n'est pas visible et deux petites bosses protubérantes sont présentes sur l'abdomen. Cette description est semblable à celle de l'espèce Oscinella frit, qui appartient à la famille des Chloropidae dont certaines espèces ont des larves qui peuvent faire des dégâts sur diverses céréales (Dajoz, 2010). Des insectes adultes appartenant à cette famille ont également été collectés au moyen des pièges sur notre parcelle expérimentale.

Quant aux larves des Lépidoptères, l'observation au laboratoire a permis de les classer comme appartenant à la famille des Pyralidae. En effet, selon Appert et Deuse (1988), les larves appartenant à cette famille sont majoritairement reconnues comme foreuses des tiges de graminées; elles sont glabres et peuvent se mouvoir à reculons. L'autre type de larve est ponctué dorsalement de noir et porte de courtes épines dispersées le long du corps. Cette morphologie est proche de celle des chenilles foreuses de tiges appartenant à la famille des Crambidae, spécifiquement au genre Chilo sp. (Polaszek et Delvare, 2000). En plus de ces auteurs, 
Brénière (1969) a également signalé des espèces de foreurs des tiges de ce genre sur le riz en Afrique francophone et à Madagascar.

La forme et l'importance des dégâts causés par les insectes foreurs des tiges selon l'âge des plants de riz NERICA montre que toutes les variétés de NERICA sont attaquées par les insectes foreurs de tiges, et que l'importance des dégâts et de leurs agents responsables varie en fonction de l'âge des plants et des variétés. Les dégâts observés (cœur mort et panicule blanche) sont le résultat du prélèvement de la nourriture à l'intérieur de la tige par les larves de Diptères et de Lépidoptères foreurs (Stoll, 2002).

L'expression des attaques des foreurs des tiges commence au stade de tallage et d'initiation paniculaire (49-56 jours) respectivement pour les variétés NERICA 11 et NERICA 14, et au stade d'épiaison pour les autres variétés ; c'est donc bien avant que les précautions doivent être prises. Le nombre de plants endommagés devient important à partir du stade d'épiaison 70 jours après le semis pour les cœurs morts, et de maturation 91 jours après le semis pour les panicules blanches. Ces résultats sont semblables à ceux obtenus en Afrique de l'Ouest par Brénière (1969).

La présence des cœurs morts jusqu'à la maturation s'explique par le fait que le tallage peut se poursuivre jusqu'à la floraison et donner des talles qui arriveront tardivement en production (Dobelmann, 1976a); ainsi, ces talles tardives sont exposées aux attaques d'insectes foreurs des tiges.

Par ailleurs, l'analyse montre aussi que 92\% de cœurs morts sont causés par les larves de Lépidoptères foreuses de tiges contre $8 \%$ dus aux larves de Diptères foreuses de tiges. Quant aux panicules blanches, 100\% de ces dégâts sont dus aux larves de Lépidoptères foreuses de tiges. Il ressort de ces analyses que les Diptères foreurs de tiges se sont attaqués aux plants de riz NERICA uniquement durant la phase végétative, alors que les attaques des Lépidoptères foreurs des tiges se sont étendues sur toutes les phases de croissance du riz. Ainsi, les Lépidoptères foreurs de tiges sont les principaux responsables des dégâts (cœurs morts et panicules blanches) observés sur les variétés de types NERICA cultivées à Franceville. Cette affirmation rejoint celle de Brink et Belay (2006).

Suite aux attaques de ces insectes au stade végétatif des plants (cœurs morts), la plante réagit par une émission de talles de remplacement qui sont à leur tour attaquées; cela entraîne une diminution du nombre de panicules. Par contre, la conséquence spécifique des attaques des larves de Lépidoptères foreurs de tiges au stade reproductif (panicules blanches) est une perte de récolte en poids sans réduction notable du nombre de panicules (Polaszek et Delvare, 2000).

\section{Estimation du niveau d'infestation des foreurs de tiges}

Les niveaux d'infestation des foreurs des tiges sur les différentes variétés de riz NERICA expérimentées sont présentés dans le Tableau 3. L'analyse de la variance indique qu'il y a une différence significative entre les variétés au seuil de $5 \%(\mathrm{P}=0,014)$. La variété NERICA 14 est la plus infestée avec un pourcentage de foreurs de tiges de 4,24\%; la variété NERICA 1 est la moins infestée $(1,77 \%)$. Dans l'ensemble, les niveaux d'infestation n'ont pas dépassé $5 \%$ et le taux moyen n'a pas excédé $3 \%$. La pression d'insectes foreurs des tiges est relativement faible. Ce faible taux d'infestation peut s'expliquer soit par la précocité des variétés NERICA, soit par la méconnaissance de la culture comme nouvel aliment pour ces insectes ou à cause des conditions climatiques certainement peu favorables à ces insectes. Ce 
résultat est comparable à celui de Gaoussou (2008). Les insectes de la famille des Diopsidae, reconnus comme foreurs de tiges en rizières, ne sont ici apparus qu'au stade de maturation, alors que d'autres auteurs (Brenière, 1969; Appert et Deuse, 1988 ; Polaszeck et Delvare, 2000) les signalent dès la phase de croissance du riz.

Elevage des larves d'insectes foreurs de tiges

En plus des adultes des Lépidoptères foreurs des tiges ayant émergé des chenilles placées dans les bocaux d'élevage, un autre insecte a fait son apparition. L'identification de cet insecte a révélé qu'il s'agit d'un Hyménoptère de la famille des Braconidae, classée comme parasite d'insectes variés. Dans notre cas, il s'agit d'un parasitoïde des chenilles foreuses des tiges. Ce résultat est en accord avec ceux de Polaszek et Delvare (2000).

\section{Chenilles défoliatrices collectées à la main} Description des larves et évolution des dégâts

Deux types de chenilles défoliatrices ont été collectés sur les variétés de riz NERICA. L'une a directement été capturée sur les feuilles et l'autre dans des enveloppes de feuilles de riz enroulées sous forme de tubes. La morphologie du type de chenilles défoliatrices est semblable à celle des larves des Lépidoptères appartenant soit à la famille des Arctiidae ou à celle des Nymphalidae (Appert et Deuse, 1988).

Le comportement et le type de dégâts dus à la chenille défoliatrice ressemblent à ceux des larves des familles de Tortricidae et de Gelechiidae. Des espèces appartenant à cette dernière famille ont été signalées sur le riz au Cameroun (Brenière, 1969).

L'analyse des données portant sur le nombre moyen de chenilles défoliatrices collectées selon l'âge des plants de riz, montre que les chenilles capturées pendant 70 jours dans les enveloppes de feuilles sont plus abondantes (17 individus) que celles capturées directement sur les feuilles. Elles ont été observées entre le $49^{\mathrm{e}}$ et le $70^{\mathrm{e}}$ jour après le semis du riz. Cette période correspond aux stades d'initiation paniculaire, de montaison et d'épiaison- floraison. Les pressions les plus élevées sont observées entre le $63^{\mathrm{e}}$ et le $70^{\mathrm{e}}$ jour, jours qui correspondent aux stades de montaison et d'épiaison-floraison, autrement dit à la phase reproductive du riz.

\section{Estimation du niveau d'infestation des chenilles défoliatrices}

L'analyse des données sur le niveau d'infestation des chenilles défoliatrices par variétés, calculés sur la base de la superficie occupée par les poquets non détruits (Tableau 4) montre que, sur le terrain expérimental, la densité des chenilles était globalement de 1,16 larves $/ \mathrm{m}^{2}$. La variété NERICA $12 \quad(2,26$ larves $/ \mathrm{m}^{2}$ ) a été la plus visitée au contraire de la variété NERICA 14 (0,55 larves/m²). Il n'y a aucune différence significative entre les autres variétés au seuil de $5 \%$.

\section{Les pucerons des racines : identification et dégâts}

Les pucerons (aptères et ailés) capturés sur les racines de riz NERICA ont été imprévisiblement découverts après déracinement d'une touffe de riz qui se caractérisait par une perte de turgescence foliaire et un retard de croissance (chétif). Ces caractéristiques ont été observées sur toutes les variétés de riz NERICA. L'identification de quelques pucerons ailés capturés avant leurs envols (après déracinement), a permis de classer ces derniers comme étant de la famille des Pemphigidae.

Les pucerons sur les racines de riz ont été continuellement observés depuis l'âge de 70 jours après le semis (épiaison-floraison), jusqu'à la récolte du riz. Cela signifie que ces ravageurs peuvent causer d'énormes pertes sur le rendement. 
L'évaluation du niveau des dégâts causés par les pucerons des racines sur la croissance du riz NERICA est présentée dans le Tableau 5. L'analyse des données du Tableau 5 montre que toutes les variétés de riz NERICA étudiées ont été infestées par les pucerons des racines et ont toutes présenté les symptômes d'infestations dus à ces derniers. Le taux d'infestation varie de $15,08 \%$ pour NERICA 14 à 34,07\% pour NERICA 12. La variété NERICA 12 est la plus menacée avec un taux d'infestation élevé, alors que les variétés NERICA 17, NERICA 11, NERICA 1 et NERICA 14 présentent les niveaux des dégâts les moins importants.

\section{Estimation des pertes de la biomasse végétative fraîche}

L'analyse des résultats (Tableau 6) sur l'estimation des pertes de la biomasse végétative fraîche montre que les attaques des insectes et des oiseaux sur le riz ont causé une perte de la masse végétative $(55,74 \%)$. Ces pertes sont énormes chez toutes les variétés. L'analyse de la variance montre qu'il n'y a pas de différence significative entre les variétés au seuil $5 \%(\mathrm{P}=0,392)$. A partir des résultats obtenus dans cette étude, on ne peut pas tirer des conclusions définitives sur les populations des insectes concernés. Il conviendra de refaire cette étude de façon à disposer d'une base plus large pour arriver à des conclusions acceptables.

L'étude complémentaire de la corrélation entre le niveau de population et les dégâts causés est aussi nécessaire pour développer un système de prévision des attaques. Pour cela, il faut d'abord identifier les principaux ravageurs de la région et évaluer leur impact économique sur la culture. Ensuite, on adoptera une approche pour développer une méthode de lutte intégrée pour la culture.

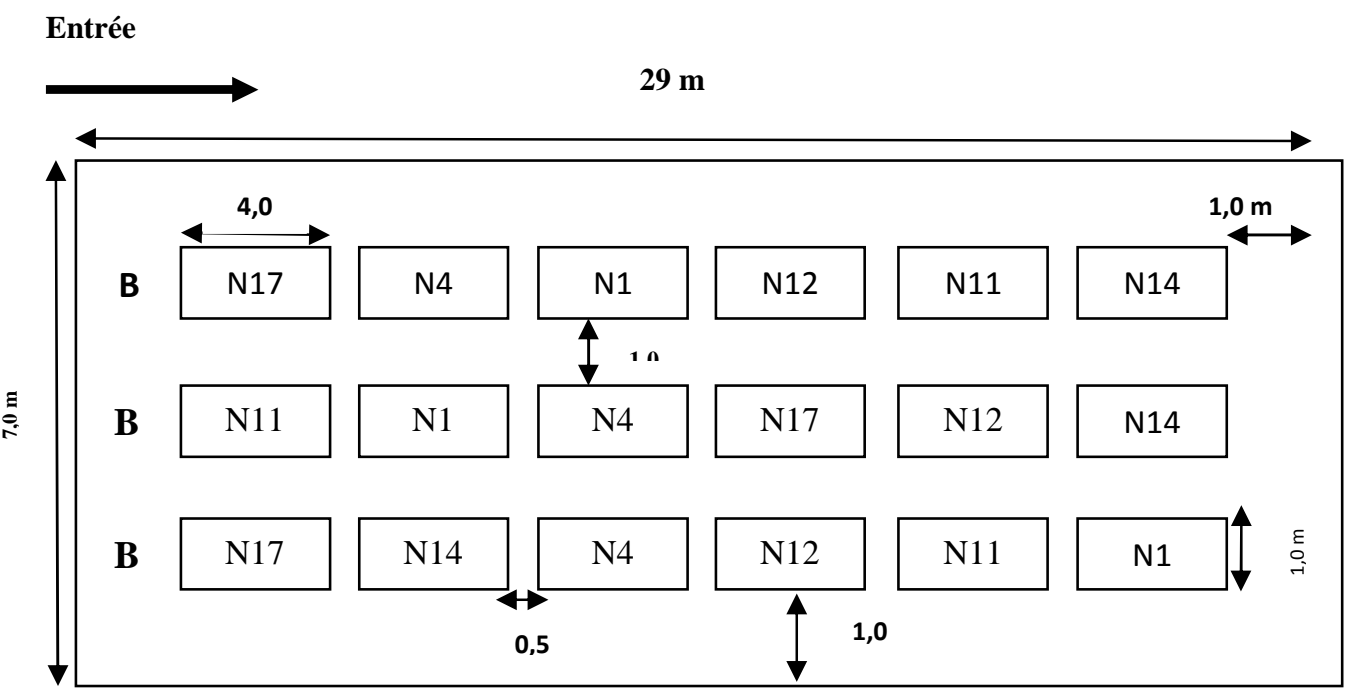

Figure 2: Dispositif expérimental (B1 à B3: Blocs ou répétitions; N1 à N17: variétés. de riz NERICA). 
P. ONDO OVONO et al. / Int. J. Biol. Chem. Sci. 8(1): 218-236, 2014
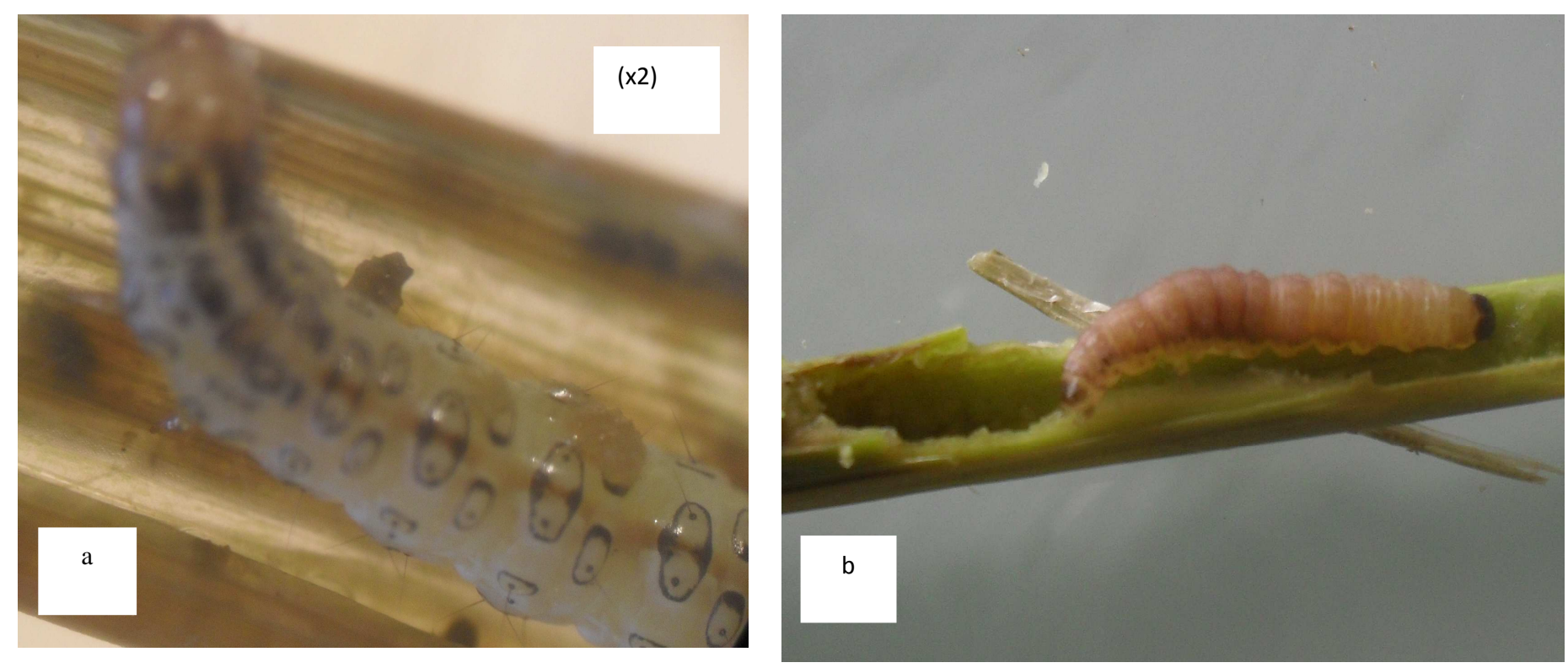

Figure 3: Deux types de chenilles (a et b) observées après la dissection des tiges. 
Tableau 1: Nombre total moyen d'insectes collectés pendant toute la période par parcelle à Franceville sur les variétés de riz NERICA à l'aide des trois types de pièges (PS, AJ et CF)

\begin{tabular}{|c|c|c|c|c|c|c|c|c|c|c|c|c|c|c|c|c|c|c|c|c|c|c|c|c|c|c|c|}
\hline \multirow{3}{*}{ Ordres } & \multirow{3}{*}{ Familles } & \multicolumn{26}{|c|}{ Nombre total d'insectes collectés par variété et par piège } \\
\hline & & \multicolumn{4}{|c|}{ NERICA 1} & \multicolumn{4}{|c|}{ NERICA 4} & \multicolumn{4}{|c|}{ NERICA 11} & \multicolumn{4}{|c|}{ NERICA 12} & \multicolumn{4}{|c|}{ NERICA 14} & \multicolumn{4}{|c|}{ NERICA 17} & \multirow{2}{*}{ 吾 } & \multirow{2}{*}{$\%$} \\
\hline & & PS & $\mathbf{A J}$ & $\mathbf{C F}$ & Tot & PS & $\mathbf{A J}$ & $\mathbf{C F}$ & Tot & PS & $\mathbf{A J}$ & $\mathbf{C F}$ & Tot & PS & $\mathbf{A J}$ & $\mathbf{C F}$ & Tot & PS & $\mathbf{A J}$ & $\mathbf{C F}$ & Tot & PS & $\mathbf{A J}$ & $\mathbf{C F}$ & Tot & & \\
\hline \multirow{3}{*}{ Orthoptera } & Grillidae & 5 & 0 & 0 & 5 & 4 & 0 & 0 & 4 & 3 & 0 & 0 & 3 & 4 & 0 & 0 & 4 & 2 & 0 & 0 & 2 & 5 & 0 & 0 & 5 & 23 & 2,40 \\
\hline & Tettigoniidae & 0 & 2 & 1 & 3 & 0 & 1 & 3 & 4 & 0 & 0 & 2 & 2 & 0 & 0 & 1 & 1 & 0 & 2 & 1 & 3 & 0 & 3 & 0 & 3 & 16 & 1,67 \\
\hline & Sauteriaux* & 27 & 0 & 1 & 28 & 35 & 0 & 2 & 37 & 29 & 0 & 2 & 31 & 24 & 0 & 2 & 26 & 24 & 0 & 0 & 24 & 30 & 0 & 3 & 33 & 179 & 18,67 \\
\hline \multicolumn{2}{|c|}{ Sous total 1} & 32 & 2 & 2 & 36 & 39 & 1 & 5 & 45 & 32 & $\mathbf{0}$ & 4 & 36 & 28 & $\mathbf{0}$ & 3 & 31 & 26 & 2 & 1 & 29 & 35 & 3 & 3 & 41 & 218 & 22,73 \\
\hline \multirow{5}{*}{ Hymenoptera } & Formicidae & 1 & 1 & 0 & 2 & 1 & 0 & 0 & 1 & 2 & 1 & 1 & 4 & 4 & 1 & 0 & 5 & 4 & 1 & 0 & 5 & 3 & 4 & 0 & 7 & 24 & 2,50 \\
\hline & Apidae & 1 & 3 & 0 & 4 & 1 & 3 & 0 & 4 & 0 & 0 & 0 & $\mathbf{0}$ & 0 & 1 & 0 & 1 & 0 & 3 & 0 & 3 & 0 & 3 & 0 & 3 & 15 & 1,56 \\
\hline & Vespidae & 2 & 4 & 0 & 6 & 1 & 3 & 0 & 4 & 0 & 1 & 0 & 1 & 0 & 4 & 0 & 4 & 0 & 4 & 0 & 4 & 1 & 3 & 0 & 4 & 23 & 2,40 \\
\hline & Elasmidae & 0 & 0 & 0 & 0 & 0 & 0 & 0 & 0 & 0 & 0 & 0 & 0 & 0 & 0 & 0 & $\mathbf{0}$ & 0 & 0 & 0 & 0 & 0 & 0 & 0 & 0 & 0 & 0,00 \\
\hline & Sphecidae & 0 & 0 & 0 & 0 & 0 & 0 & 0 & 0 & 0 & 0 & 0 & 0 & 0 & 1 & 0 & 1 & 0 & 1 & 0 & 1 & 0 & 0 & 0 & $\mathbf{0}$ & 2 & 0,21 \\
\hline \multicolumn{2}{|c|}{ Sous total 2} & 4 & 8 & $\mathbf{0}$ & 12 & 3 & 6 & $\mathbf{0}$ & 9 & 2 & 2 & 1 & 5 & 4 & 7 & $\mathbf{0}$ & 11 & 4 & 9 & $\mathbf{0}$ & 13 & 4 & 10 & $\mathbf{0}$ & 14 & 64 & 6,67 \\
\hline \multirow{7}{*}{ Diptera } & Agromyzidae & 2 & 7 & 0 & 9 & 1 & 4 & 0 & 5 & 1 & 4 & 0 & 5 & 3 & 2 & 0 & 5 & 2 & 5 & 0 & 7 & 3 & 5 & 0 & 8 & 39 & 4,07 \\
\hline & Chloropidae & 1 & 1 & 0 & 2 & 1 & 0 & 0 & 1 & 1 & 0 & 0 & 1 & 2 & 0 & 0 & 2 & 1 & 1 & 0 & 2 & 1 & 1 & 0 & 2 & 10 & 1,04 \\
\hline & Calliphoridae & 1 & 8 & 0 & 9 & 2 & 3 & 0 & 5 & 1 & 6 & 0 & 7 & 1 & 3 & 0 & 4 & 1 & 6 & 0 & 7 & 1 & 2 & 0 & 3 & 35 & 3,65 \\
\hline & Tachinidae & 1 & 1 & 2 & 4 & 1 & 2 & 0 & 3 & 1 & 1 & 1 & 3 & 2 & 2 & 1 & 5 & 2 & 3 & 0 & 5 & 0 & 1 & 0 & 1 & 21 & 2,19 \\
\hline & Stratiomydae & 0 & 0 & 0 & $\mathbf{0}$ & 0 & 4 & 0 & 4 & 0 & 0 & 0 & 0 & 0 & 1 & 0 & 1 & 0 & 1 & 0 & 1 & 0 & 0 & 0 & $\mathbf{0}$ & 6 & 0,63 \\
\hline & Syrphidae & 0 & 2 & 0 & 2 & 0 & 2 & 0 & 2 & 0 & 1 & 0 & 1 & 0 & 1 & 0 & 1 & 0 & 2 & 0 & 2 & 0 & 3 & 0 & 3 & 11 & 1,15 \\
\hline & Diopsidae & 0 & 0 & 0 & $\mathbf{0}$ & 0 & 0 & 0 & $\mathbf{0}$ & 0 & 0 & 0 & $\mathbf{0}$ & 0 & 0 & 0 & $\mathbf{0}$ & 0 & 0 & 0 & $\mathbf{0}$ & 0 & 0 & 1 & 1 & 1 & 0,10 \\
\hline \multicolumn{2}{|c|}{ Sous total 3} & 5 & 19 & 2 & 26 & 5 & 15 & $\mathbf{0}$ & 20 & 4 & 12 & 1 & 17 & 8 & 9 & 1 & 18 & 6 & 18 & $\mathbf{0}$ & 24 & 5 & 12 & 1 & 18 & 123 & 12,83 \\
\hline \multirow{4}{*}{ Coleoptera } & Coccinellidae & 0 & 0 & 0 & $\mathbf{0}$ & 0 & 0 & 0 & $\mathbf{0}$ & 0 & 0 & 0 & $\mathbf{0}$ & 1 & 1 & 0 & 2 & 1 & 3 & 0 & 4 & 0 & 0 & 0 & $\mathbf{0}$ & 6 & 0,63 \\
\hline & Lampyridae & 0 & 0 & 0 & $\mathbf{0}$ & 0 & 0 & 0 & $\mathbf{0}$ & 0 & 0 & 0 & $\mathbf{0}$ & 0 & 0 & 0 & $\mathbf{0}$ & 0 & 0 & 0 & $\mathbf{0}$ & 0 & 0 & 0 & $\mathbf{0}$ & $\mathbf{0}$ & 0,00 \\
\hline & Chrysomelidae & 0 & 3 & 3 & 6 & 0 & 0 & 3 & 3 & 0 & 2 & 2 & 4 & 1 & 1 & 4 & 6 & 0 & 0 & 5 & 5 & 0 & 4 & 5 & 9 & 33 & 3,44 \\
\hline & Carabidae & 1 & 0 & 0 & 1 & 0 & 0 & 0 & $\mathbf{0}$ & 0 & 0 & 0 & $\mathbf{0}$ & 0 & 0 & 0 & $\mathbf{0}$ & 1 & 0 & 0 & 1 & 1 & 0 & 0 & 1 & 3 & 0,31 \\
\hline \multicolumn{2}{|c|}{ Sous total 4} & 1 & 3 & 3 & 7 & $\mathbf{0}$ & $\mathbf{0}$ & 3 & 3 & $\mathbf{0}$ & 2 & 2 & 4 & 2 & 2 & 4 & 8 & 2 & 3 & 5 & 10 & 1 & 4 & 5 & 10 & 42 & 4,38 \\
\hline \multirow{5}{*}{ Lepidoptera } & Noctuidae & 2 & 2 & 0 & 4 & 1 & 3 & 0 & 4 & 1 & 2 & 0 & 3 & 2 & 2 & 0 & 4 & 0 & 2 & 0 & 2 & 0 & 5 & 0 & 5 & 22 & 2,29 \\
\hline & Pyralidae & 0 & 4 & 0 & 4 & 1 & 10 & 0 & 11 & 2 & 6 & 0 & 8 & 1 & 6 & 0 & 7 & 2 & 10 & 0 & 12 & 0 & 6 & 0 & 6 & 48 & 5,01 \\
\hline & Arctiidae & 0 & 0 & 0 & $\mathbf{0}$ & 0 & 0 & 1 & 1 & 0 & 0 & 0 & $\mathbf{0}$ & 0 & 0 & 1 & 1 & 0 & 0 & 0 & $\mathbf{0}$ & 0 & 0 & 1 & 1 & 3 & 0,31 \\
\hline & Nymphalidae & 0 & 3 & 0 & 3 & 0 & 0 & 0 & $\mathbf{0}$ & 0 & 2 & 0 & 2 & 0 & 1 & 0 & 1 & 0 & 0 & 0 & 0 & 0 & 1 & 0 & 1 & 7 & 0,73 \\
\hline & Non identifié & 0 & 0 & 0 & o & 4 & 0 & 0 & 4 & 2 & 0 & 0 & 2 & 3 & 0 & 0 & 3 & 2 & 0 & 0 & 2 & 2 & 0 & 0 & 2 & 13 & 1,36 \\
\hline
\end{tabular}


P. ONDO OVONO et al. / Int. J. Biol. Chem. Sci. 8(1): 218-236, 2014

\begin{tabular}{|c|c|c|c|c|c|c|c|c|c|c|c|c|c|c|c|c|c|c|c|c|c|c|c|c|c|c|c|}
\hline \multicolumn{2}{|c|}{ Sous total 5} & 2 & 9 & $\mathbf{0}$ & 11 & 6 & 13 & 1 & 20 & 5 & 10 & $\mathbf{0}$ & 15 & 6 & 9 & 1 & 16 & 4 & 12 & $\mathbf{0}$ & 16 & 2 & 12 & 1 & 15 & 93 & 9,70 \\
\hline \multirow{15}{*}{ Hemiptera } & Reduviidae & 0 & 0 & 1 & 1 & 0 & 1 & 0 & 1 & 0 & 1 & 0 & 1 & 0 & 0 & 0 & $\mathbf{0}$ & 0 & 0 & 0 & $\mathbf{0}$ & 0 & 1 & 0 & 1 & 4 & 0,42 \\
\hline & Lygaeidae & 0 & 0 & 0 & 0 & 0 & 0 & 0 & o & 0 & 0 & 0 & $\mathbf{0}$ & 0 & 0 & 0 & $\mathbf{0}$ & 1 & 0 & 0 & 1 & 0 & 0 & 0 & $\mathbf{0}$ & 1 & 0,10 \\
\hline & Coreidae & 0 & 0 & 0 & 0 & 0 & 0 & 0 & o & 0 & 0 & 0 & 0 & 0 & 0 & 0 & o & 0 & 0 & 0 & 0 & 0 & 0 & 0 & o & 0 & 0,00 \\
\hline & Alydidae & 1 & 2 & 0 & 3 & 0 & 1 & 0 & 1 & 0 & 0 & 0 & o & 0 & 1 & 0 & 1 & 1 & 1 & 0 & 2 & 1 & 1 & 0 & 2 & 9 & 0,94 \\
\hline & Pyrrhocoridae & 0 & 0 & 0 & 0 & 0 & 0 & 0 & 0 & 0 & 0 & 0 & 0 & 0 & 0 & 0 & 0 & 0 & 0 & 0 & 0 & 0 & 0 & 0 & 0 & 0 & 0,00 \\
\hline & Pentatomidae & 1 & 0 & 0 & 1 & 0 & 1 & 0 & 1 & 1 & 0 & 0 & 1 & 0 & 0 & 0 & 0 & 0 & 1 & 0 & 1 & 0 & 0 & 0 & 0 & 4 & 0,42 \\
\hline & Cicadellidae & 0 & 6 & 1 & 7 & 0 & 4 & 0 & 4 & 0 & 3 & 0 & 3 & 0 & 3 & 1 & 4 & 0 & 6 & 3 & 9 & 0 & 5 & 0 & 5 & 32 & 3,34 \\
\hline & Ricaniidae & 0 & 0 & 0 & $\mathbf{0}$ & 0 & 0 & 0 & 0 & 0 & 0 & 0 & 0 & 0 & 0 & 0 & 0 & 0 & 0 & 0 & 0 & 0 & 0 & 0 & 0 & $\mathbf{0}$ & 0,00 \\
\hline & Cercopidae & 0 & 0 & 0 & 0 & 0 & 0 & 0 & $\mathbf{0}$ & 0 & 0 & 0 & 0 & 0 & 0 & 0 & 0 & 0 & 1 & 0 & 1 & 0 & 1 & 0 & 1 & 2 & 0,21 \\
\hline & Scutelleridae & 0 & 0 & 0 & $\mathbf{0}$ & 0 & 0 & 0 & $\mathbf{0}$ & 0 & 0 & 0 & $\mathbf{0}$ & 0 & 0 & 0 & $\mathbf{0}$ & 0 & 0 & 0 & $\mathbf{0}$ & 0 & 0 & 0 & $\mathbf{0}$ & $\mathbf{0}$ & 0,00 \\
\hline & Delphacidae & 0 & 4 & 1 & 5 & 0 & 2 & 0 & 2 & 0 & 2 & 0 & 2 & 0 & 1 & 1 & 2 & 0 & 5 & 2 & 7 & 0 & 3 & 0 & 3 & 21 & 2,19 \\
\hline & Aphididae & 0 & 52 & 0 & 52 & 0 & 51 & 0 & 51 & 0 & 52 & 0 & 52 & 0 & 52 & 1 & 53 & 0 & 55 & 1 & 56 & 0 & 51 & 0 & 51 & 315 & 32,85 \\
\hline & Membracidae & 0 & 0 & 0 & $\mathbf{0}$ & 0 & 1 & 0 & 1 & 0 & 0 & 0 & $\mathbf{0}$ & 0 & 0 & 0 & $\mathbf{0}$ & 0 & 0 & 0 & $\mathbf{0}$ & 0 & 0 & 0 & $\mathbf{0}$ & 1 & 0,10 \\
\hline & Lophopidae & 0 & 0 & 2 & 2 & 0 & 0 & 1 & 1 & 0 & 0 & 0 & $\mathbf{0}$ & 0 & 0 & 0 & $\mathbf{0}$ & 0 & 1 & 0 & 1 & 0 & 0 & 1 & 1 & 5 & 0,52 \\
\hline & Plataspidae & 0 & 0 & 0 & 0 & 0 & 0 & 1 & 1 & 0 & 0 & 0 & 0 & 0 & 0 & $\mathbf{0}$ & $\mathbf{0}$ & 0 & 0 & 0 & $\mathbf{0}$ & 0 & 0 & 0 & $\mathbf{0}$ & 1 & 0,10 \\
\hline \multicolumn{2}{|c|}{ Sous total 6} & 2 & 64 & 5 & 71 & $\mathbf{0}$ & 61 & 2 & 63 & 1 & 58 & $\mathbf{0}$ & 59 & $\mathbf{0}$ & 57 & 3 & 60 & 2 & 70 & 6 & 78 & 1 & 62 & 1 & 64 & 395 & 41,19 \\
\hline \multirow{2}{*}{ Dictyoptera } & Blattidae & 0 & 0 & 0 & $\mathbf{0}$ & 0 & 1 & $\mathbf{0}$ & 1 & 0 & 0 & 0 & $\mathbf{0}$ & 0 & 2 & 0 & 2 & 0 & 3 & 0 & 3 & 0 & 2 & 0 & 2 & 8 & 0,83 \\
\hline & Mantidae & 0 & 0 & 0 & $\mathbf{0}$ & 0 & 0 & $\mathbf{0}$ & $\mathbf{0}$ & 0 & 0 & 0 & $\mathbf{0}$ & 0 & 0 & 0 & $\mathbf{0}$ & 0 & 0 & 0 & $\mathbf{0}$ & 0 & 0 & 0 & 0 & 0 & 0,00 \\
\hline \multicolumn{2}{|c|}{ Sous total 7} & $\mathbf{0}$ & $\mathbf{0}$ & $\mathbf{0}$ & $\mathbf{0}$ & $\mathbf{0}$ & 1 & $\mathbf{0}$ & 1 & $\mathbf{0}$ & $\mathbf{0}$ & $\mathbf{0}$ & $\mathbf{0}$ & $\mathbf{0}$ & 2 & $\mathbf{0}$ & 2 & $\mathbf{0}$ & 3 & $\mathbf{0}$ & 3 & $\mathbf{0}$ & 2 & $\mathbf{0}$ & 2 & 8 & $\mathbf{0 , 8 3}$ \\
\hline \multirow{2}{*}{ Nevroptera } & Chrysopidae & 0 & 0 & 0 & 0 & 0 & 1 & $\mathbf{0}$ & 1 & 0 & 0 & 0 & $\mathbf{0}$ & 0 & 0 & 0 & 0 & 0 & 1 & 0 & 1 & 0 & 2 & 0 & 2 & 4 & 0,42 \\
\hline & Hemerobiidae & 0 & 0 & 0 & 0 & 0 & 0 & 0 & 0 & 0 & 1 & 0 & 1 & 0 & 0 & 0 & 0 & 0 & 0 & 0 & 0 & 0 & 0 & 0 & o & 1 & 0,10 \\
\hline \multicolumn{2}{|c|}{ Sous total 8} & $\mathbf{0}$ & $\mathbf{0}$ & $\mathbf{0}$ & $\mathbf{0}$ & $\mathbf{0}$ & 1 & $\mathbf{0}$ & 1 & $\mathbf{0}$ & 1 & $\mathbf{0}$ & 1 & $\mathbf{0}$ & $\mathbf{0}$ & $\mathbf{0}$ & $\mathbf{0}$ & $\mathbf{0}$ & 1 & $\mathbf{0}$ & 1 & $\mathbf{0}$ & 2 & $\mathbf{0}$ & 2 & 5 & 0,52 \\
\hline Thysanoptera & Thripidae & 0 & 0 & 2 & 2 & 0 & 0 & 2 & 2 & 0 & 0 & 1 & 1 & 0 & $\mathbf{0}$ & 2 & 2 & 0 & $\mathbf{0}$ & 2 & 2 & 0 & 0 & 2 & 2 & 11 & 1,15 \\
\hline \multicolumn{2}{|c|}{ Sous total 9} & $\mathbf{0}$ & $\mathbf{0}$ & 2 & 2 & $\mathbf{0}$ & $\mathbf{0}$ & 2 & 2 & $\mathbf{0}$ & $\mathbf{0}$ & 1 & 1 & $\mathbf{0}$ & $\mathbf{0}$ & 2 & 2 & $\mathbf{0}$ & $\mathbf{0}$ & 2 & 2 & $\mathbf{0}$ & $\mathbf{0}$ & 2 & 2 & 11 & 1,15 \\
\hline \multicolumn{2}{|c|}{ Total } & 46 & 105 & 14 & 165 & 53 & 98 & 13 & 164 & 44 & 85 & 9 & 138 & 48 & 86 & 14 & 148 & 44 & 118 & 14 & 176 & 48 & 107 & 13 & 168 & 959 & \\
\hline \multicolumn{2}{|c|}{$\%$} & 4,80 & 10,95 & 1,46 & 17,21 & 5,53 & 10,22 & 1,36 & 17,10 & 4,59 & 8,86 & 0,94 & 14,39 & 5,01 & 8,97 & 1,46 & 15,43 & 4,59 & 12,30 & 1,46 & 18,35 & 5,01 & 11,16 & 1,36 & 17,52 & & 100,00 \\
\hline
\end{tabular}

PS : piège au sol ; AJ : pièges aériens aux assiettes (ou bacs) jaunes ; CF : capture au filet fauchoir. 
Tableau 2: Nombre d'insectes ravageurs collectés durant le cycle cultural des variétés de riz NERICA.

\begin{tabular}{lcc}
\hline Variétés & Nombre d'insectes & $\%$ \\
\hline NERICA 14 & 142 & $18 \mathrm{a}$ \\
NERICA 17 & 141 & $18 \mathrm{a}$ \\
NERICA 1 & 135 & $17 \mathrm{ab}$ \\
NERICA 4 & 135 & $17 \mathrm{ab}$ \\
NERICA 12 & 122 & $15 \mathrm{~b}$ \\
NERICA 11 & 120 & $15 \mathrm{~b}$ \\
\hline
\end{tabular}

Les valeurs suivies d'une même lettre ne sont pas significativement différentes selon le test de Duncan au seuil 5\%.

Tableau 3 : Taux d'infestation (\% de plants infectés) des foreurs des tiges par variétés de NERICA.

\begin{tabular}{lc}
\hline Variétés & Taux d'infestation des foreurs des tiges $(\mathbf{C M + P B})$ \\
\hline NERICA 14 & $4,24 \mathrm{a}$ \\
NERICA 17 & $2,90 \mathrm{ab}$ \\
NERICA 11 & $2,35 \mathrm{ab}$ \\
NERICA 12 & $2,10 \mathrm{ab}$ \\
NERICA 4 & $1,89 \mathrm{ab}$ \\
NERICA 1 & $1,77 \mathrm{~b}$ \\
\hline
\end{tabular}

$\mathrm{CM}$ : cœurs morts ; $\mathrm{PB}$ : panicules blanches ; les valeurs suivies d'une même lettre ne sont pas significativement différentes selon le test de DUNCAN au seuil 5\%.

Tableau 4 : Niveau d'infestation des chenilles défoliatrices par variétés de NERICA.

\begin{tabular}{lc}
\hline Variétés & Nombre de chenilles $/ \mathbf{m}^{2}$ \\
\hline NERICA 12 & $2,26 \mathrm{a}$ \\
NERICA 4 & $1,19 \mathrm{ab}$ \\
NERICA 11 & $1,15 \mathrm{ab}$ \\
NERICA 17 & $0,96 \mathrm{ab}$ \\
NERICA 1 & $0,84 \mathrm{ab}$ \\
NERICA 14 & $0,55 \mathrm{~b}$ \\
\hline
\end{tabular}

Les valeurs suivies d'une même lettre ne sont pas significativement différentes selon le test de DUNCAN au seuil 5\%. 
Tableau 5 : Niveau des dégâts dus aux pucerons des racines par variétés de NERICA.

\begin{tabular}{lc}
\hline Variétés & Taux d'infestation $(\%)$ \\
\hline NERICA 12 & $34,07 \mathrm{a}$ \\
NERICA 4 & $24,51 \mathrm{ab}$ \\
NERICA 17 & $17,46 \mathrm{~b}$ \\
NERICA 11 & $17,20 \mathrm{~b}$ \\
NERICA 1 & $16,63 \mathrm{~b}$ \\
NERICA 14 & $15,08 \mathrm{~b}$ \\
\hline Les valeurs suivies d'une même lettre ne sont pas significativement différentes selon le test \\
de Duncan au seuil 5\%.
\end{tabular}

Tableau 6: Pertes de la biomasse végétative aérienne fraîche par variétés de riz NERICA.

\begin{tabular}{lc}
\hline Variétés & Taux d'infestation $(\%)$ \\
\hline NERICA 17 & $63,94 \mathrm{a}$ \\
NERICA 12 & $59,49 \mathrm{a}$ \\
NERICA 11 & $57,32 \mathrm{a}$ \\
NERICA 1 & $55,50 \mathrm{a}$ \\
NERICA 14 & $53,15 \mathrm{a}$ \\
NERICA 4 & $45,06 \mathrm{a}$ \\
\hline
\end{tabular}

Les valeurs suivies d'une même lettre ne sont pas significativement différentes selon le test de Duncan au seuil $5 \%$.

\section{Conclusion}

L'étude sur la dynamique des populations d'insectes du riz NERICA dans les conditions pédoclimatiques du Sud-Est du Gabon à Franceville, a permis de montrer que les fluctuations en nombre et en espèces sont fonction des stades phénologiques de la plante. Au total, 46 familles appartenant à 9 ordres d'insectes ont été recensés. La plus forte population s'est retrouvée dans l'ordre des Hémiptères (41,19\%), suivie des Orthoptères (22,73\%), des Diptères $(12,83 \%)$, des Lépidoptères $(9,70 \%)$, des Hyménoptères $(6,67 \%)$, des Coléoptères $(4,38 \%)$, des Thysanoptères $(1,15 \%)$, des Dictyoptères $(0,83 \%)$ et des Névroptères $(0,52 \%)$.

Les Orthoptères ont été abondamment collectés à l'aide des pièges placés au sol. Les Hyménoptères, les Diptères, les Lépidoptères et les Hémiptères ont été largement collectés dans les pièges aériens. Les Coléoptères ont été principalement collectés à l'aide du filet entomologique. Les Névroptères et les
Thysanoptères ont été uniquement collectés respectivement au moyen des pièges aériens et $\mathrm{du}$ filet entomologique.

Les insectes définis comme ravageurs ont été d'une manière générale nombreux et actifs sur le riz aux stades de tallage, d'initiation paniculaire et d'épiaison-floraison. Ils représentent $83 \%$ du total recensé. Les $17 \%$ restant sont répartis entre les auxiliaires, les saprophages et les coprophages. Les Orthoptères ont été en particulier plus importants sur la culture au stade plantule. L'abondance des insectes à un stade par rapport à un autre s'explique par les préférences alimentaires mais aussi par les conditions climatiques. La pression d'insectes foreurs des tiges a relativement été faible avec un taux moyen de dégâts de $2,54 \%$. Le taux le plus faible de dégâts a été enregistré sur le NERICA $1(1,77 \%)$ et le plus élevé sur le NERICA 14 (4,24\%). Dans l'ensemble, les variétés NERICA 1 et NERICA 11 ont présenté les taux les plus faibles d'infestation. 
La sensibilité des variétés est variable en fonction du type d'insectes. Ainsi NERICA 14 est peu atteint par les chenilles défoliatrices et les pucerons alors que le taux d'infestation par les foreuses de tiges est le plus élevé ainsi que le nombre d'insectes ravageurs recensés. Par ailleurs, les pertes de la biomasse végétative imputables aux attaques d'insectes et d'oiseaux ont été énormes sur toutes les variétés avec un taux moyen de 55,75\%.

\section{REMERCIEMENTS}

Nous remercions le Dr Daniel OBAME ONDO, ex-Directeur de l'ONADER et promoteur du projet NERICA au niveau national et MOUNGUENGUI Judicaël, Ingénieur agronome et coordinateur provincial du Haut Ogooué de l'ONADER, pour leur appui technique. Nous remercions également les techniciens de l'Institut National Supérieur d'Agronomie et de Biotechnologies pour leur appui dans le traitement statistique des données.

\section{RÉFÉRENCES}

ADRAO. 2003. NERICA en plein essor: un symbole d'espoir pour les riziculteurs africains. Abidjan, Côte d'Ivoire, 2 p. http : w.w.w. warda.org (le 03 juillet 2011).

Akintayo I, Cissé B, Zadji LD. 2008. Guide Pratique de la Culture des NERICA de Plateau. Centre du riz pour l'Afrique (ADRAO) : Cotonou, Benin ; 36 p.

Appert J, Deuse J. 1988. Insectes Nuisibles aux Cultures Vivrières et Maraîchères (Tome 1 et 2). Editions Maisonneuve et Larose : Paris, France; 105p ; 267p.

Boucher S. 2008. Les Insectes de nos Jardins. Editions Broquet : Québec, 208 p.

Brenière J. 1966. Dix années de recherche sur les ennemis $\mathrm{du}$ riz en Afrique francophone et à Madagascar. L'Agronomie Tropicale, 4: 514-519.

Brenière J. 1969. Importance des problèmes entomologiques dans le développement de la riziculture de l'Afrique de l'Ouest. L'Agronomie Tropicale, 10: 906-927.

Brink M, Belay G. 2006. Ressources Végétales de l'Afrique Tropicale 1. Céréales et Légumes Secs. Fondation PROTA, Backhuys Publishers: Wageningen, Pays-Bas.

Courtois B. 2007.Une Brève Histoire de l'Amélioration Génétique du Riz. CIRAD : France; $13 \mathrm{p}$.

Dajoz R. 2010. Dictionnaire d'Entomologie. Edition Lavoisier : Paris, France; 336 p.

Delvare G., Aberlenc HP. 1989. Les insectes d'Afrique et d'Amérique Tropicale. Clés pour la Reconnaissance des Familles: Montpellier, France; 302 p.

Dobelmann JP. 1976a. Riziculture Pratique. 1. Riz Irrigué. Techniques Vivantes. Agence de Coopération Culturelle et Technique : Paris, France; 229 p.

Dobelmann JP. 1976b. Riziculture Pratique.2. Riz Pluvial. Techniques Vivantes. Agence de Coopération Culturelle et Technique : Paris France; 131 p.

Dogbe SY. 2009. Etude sur le développement $\mathrm{du}$ sous -secteur riz au Gabon, rapport d'étude, juin 2009, 39 p.

Dupriez H, Silas N, Colin J. 2001. Champs et Jardins Saints, Lutte Intégrée. Carnets Ecologiques.12 Afrique. Editions Terres et Vie et CTA: Nivelles, Belgique; 238 p.

Gaoussou N. 2008. Evaluation multilocale de nouvelles variétés de riz en conditions de bas-fonds et irriguée de l'Ouest du Burkina-Faso. Mémoire de fin de cycle, Institut du Développement Rural : Université Polytechnique de BoboDioulasso, Burkina Faso, 83 p.

IRRI (International Rice Research Institute). 2005. Wild Rice Taxonomy. IRRI.

Itongo MT. 1998. Géomorphologie et prévision des Risques d'érosion dans la région de Franceville (Gabon). Thèse de doctorat, Université Louis Pasteur (Strasbourg) mention géographie et physique, 264 p. 
Kumar R. 1991. La Lutte Contre les Insectes Ravageurs; la Situation de l'Agriculture Africaine. Editions CTA-KARTHALA : Paris, France; $310 \mathrm{p}$.

Limoges R. 2003. Méthode de capture 2, Insectarium de Montréal. http:// www2.villemontreal.qc.ca (le 16 juin 2011).

Maganga LM. 2010. Evaluation au champ des performances agromorphologiques des variétés de riz NERICA, cultivé au sudest du Gabon. Mémoire de fin de cycle, INSAB/USTM, Franceville, 62 p.

Michel B, Bournier JP. 1997. Les Auxiliaires de Cultures Tropicales. Cirad: Montpellier, France; 88 p.

Ndayiragije A. 2006. Relations entre métabolisme des polyamines et résistance au stress salin chez le riz (Oryza sativa L.). Thèse de Doctorat en ingénierie biologique, agronomique et environnementale. Université Catholique de Louvain. Faculté d'ingénierie biologique, agronomique et environnementale, $251 \mathrm{p}$.

Nguimbi L. 2004. Rapport définitif du projet Diagnostic de la filière riz au Gabon, IRAF : Libreville, Gabon, 60 p.
Pande HK, Tran DV, That TT. 1997. Systèmes améliorés de riziculture pluviale, FAO, Rome, http://www.fao.org (le 25 juin 2011).

Polaszek A, Delvare G. 2000. Les Foreurs des Tiges de Céréales en Afrique. Importance Economique, Systématique, Ennemis Naturels et Méthodes de Lutte. CiradCTA : Toulouse, France; 534 p.

Schalbroeck JJ. 2001. Le riz: Agriculture en Afrique Tropicale. Ministère des Affaires étrangères du Commerce extérieur et de la Coopération Internationale : Bruxelles, Belgique, p.99-105.

Simon H, Richard F, Bellanger M, Denimal D, Goubert C, Jeuffrault E. 1994. La Protection des Cultures. Agriculture d'Aujourd'hui. Edition Lavoisier: Paris, France; 3.

Stoll G. 2002. Protection Naturelle des Végétaux en Zones Tropicales; Vers une Dynamique de l'Information. Edition Margraf Verlag: Weikersheim, Allemagne; $386 \mathrm{p}$.

Tréca B, Ndiaye AB, Manikowski S. 1997. Oiseaux et Déprédateurs des Cultures au Sahel. Edition John Libbey Eurotex, CTA, ORSTOM : Paris France ; 24-29. 